\title{
Topics
}

\section{Optimal Trajectory Design for Asteroid Deflection Mission}

\author{
By Mai BANDO ${ }^{1)}$ and Hiroshi YAMAKAwA ${ }^{1)}$ \\ ${ }^{1)}$ Kyoto University, Kyoto, Japan
}

(Received June 27th, 2011)

\begin{abstract}
The trajectory design associated with the deflection of potentially hazardous asteroid (PHA) is considered. At the terminal mission phase, low-thrust guidance control is used to compensate state errors and to change the intercept angle from nominal one to meet mission requirements. In this paper, the robust trajectory design using low-thrust acceleration is considered. We employ the generating functions approach proposed by Guibout and Scheeres (2004) and investigate its application to robust terminal guidance control. Our approach is illustrated through the study of the asteroid deflection mission.
\end{abstract}

Key Words: Potentially Hazardous Asteroids, Low-Thrust Trajectory Design, Asteroid Deflection

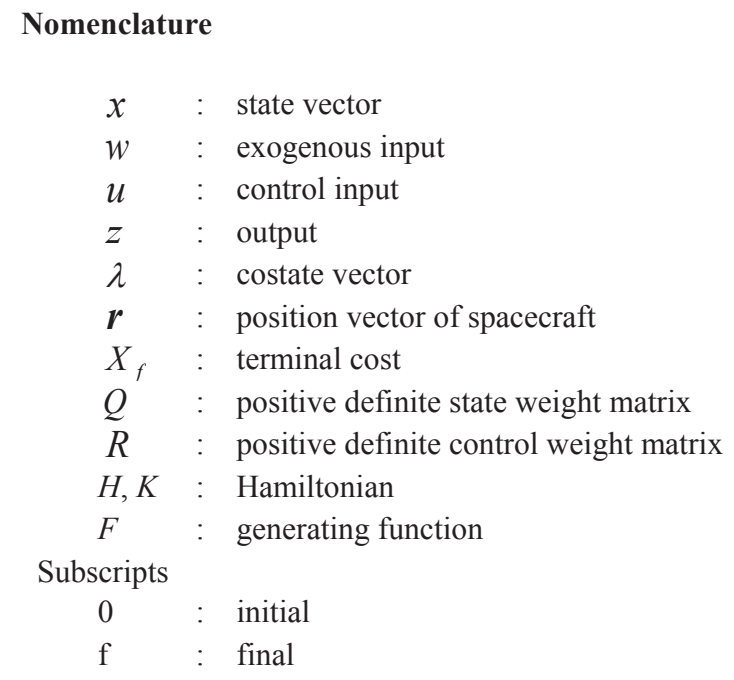

\section{Introduction}

The threat of an asteroid impacting Earth have attracted much attention recent years. Many techniques to deflect potentially hazardous asteroids (PHAs) have been proposed. ${ }^{1)}$ In many cases, only a slight perturbation is sufficient to produce the desired deflection. The objective of a kinetic impactor mission ${ }^{2)-7)}$ would be impacting the target at a sufficiently high velocity and to transfer the momentum to change the orbit. The relative velocity at impact would be determined by the specific intercept trajectory. However, it is difficult to determine exact values of the orbital elements, mass, shape and the surface structure of the target asteroids. Therefore, the robust trajectory design for kinetic impactor spacecraft, including the uncertainty and options to use the low-thrust acceleration to correct the terminal velocity vector to maximize the closest approach distance to the Earth would be necessary. Moreover, the asteroid deflection mission with a controlled intercept angle is considered to meet various mission requirements.
We assume the nominal trajectory given by Lambert solution which is obtained from orbit determination at the initial phase. Then at the terminal guidance phase, robust terminal controller is applied to compensate state errors and to change the impact angle from nominal one. Fuel requirement is critical factor to control the intercept angle. For low relative impact velocities, it is feasible to carry enough fuel onboard to significantly change the intercept angle even in the terminal phase of the mission. We derive the optimal terminal guidance law based on generating functions approach ${ }^{8)-10)}$ and analyze the performance of it.

Optimal rendezvous problem using continuous low-thrust is treated as optimal control problem. Using the property that both problems can be treated as two-point boundary value problem (TPVBP) of Hamiltonian system, we formulate the robust guidance control law to asteroid which minimize the quadratic cost under uncertainty by generating functions. The emphasis is that this approach enables us to find optimal trajectory to complicated problem not by a global search but by function evaluations. Our approach is illustrated through the study of the asteroid deflection mission.

\section{Deflection Mission Scenario}

At first stage of the mission, orbits of the spacecraft and target are assumed to be known as a result of orbit determination and the spacecraft impulsive maneuver is determined by solving Lambert's problem. During the terminal phase, observations of the target are used to compute a relative velocity and guidance control is needed to correct the terminal intercept angle and so on from onboard observations.

\section{Solution to TPVBP Using Generating Functions}

3.1. Canonical transformation of hamilton system In this section, we briefly review the theory of canonical 
transformations and generating functions in Hamiltonian dynamics. ${ }^{11)}$ Following Refs. 8) through 10), we introduce the canonical transformations between a moving initial state $(x(t), \lambda(t))$, and fixed terminal state $\left(x\left(t_{f}\right), \lambda\left(t_{f}\right)\right)$. Let $H(x, \lambda, t)$ be a Hamiltonian of the system. Suppose that there exists a canonical transformation from $(x(t), \lambda(t))$ to a new set of coordinate $\left(x\left(t_{f}\right), \lambda\left(t_{f}\right)\right)$ with Hamiltonian $K\left(x\left(t_{f}\right), \lambda\left(t_{f}\right), t\right)$. Since the latter set of coordinate are constants, i.e. $\left(x_{f}, \lambda_{f}\right)=\left(x\left(t_{f}\right), \lambda\left(t_{f}\right)\right)$, and Hamiltonian of a fixed state can be defined as zero, we set $K\left(x_{f}, \lambda_{f}, t\right)=0$. Then there exist generating functions associated with the transformations which can be written in following forms ${ }^{11)}$ :

$$
\begin{aligned}
F_{1}: \lambda_{f}= & \frac{\partial F_{1}\left(x_{f}, \lambda, t_{f} ; t\right)}{\partial x_{f}}, \quad \lambda(t)=-\frac{\partial F_{1}\left(x_{f}, \lambda, t_{f} ; t\right)}{\partial x} \\
& \frac{\partial F_{1}\left(x_{f}, \lambda, t_{f} ; t\right)}{\partial t}+H\left(x_{f},-\frac{\partial F_{1}\left(x_{f}, \lambda, t_{f} ; t\right)}{\partial x}, t\right)=0 \\
F_{2}: \lambda(t) & =\frac{\partial F_{2}\left(x_{f}, \lambda, t_{f} ; t\right)}{\partial x_{f}}, \quad x(t)=-\frac{\partial F_{2}\left(x_{f}, \lambda, t_{f} ; t\right)}{\partial \lambda} \\
- & \frac{\partial F_{2}\left(x_{f}, \lambda, t_{f} ; t\right)}{\partial t}+H\left(-\frac{\partial F_{21}\left(x_{f}, \lambda, t_{f} ; t\right)}{\partial \lambda}, \lambda, t\right)=0 \\
F_{3}: x_{f}= & -\frac{\partial F_{3}\left(\lambda_{f}, x, t_{f} ; t\right)}{\partial \lambda_{f}}, \quad \lambda(t)=-\frac{\partial F_{3}\left(\lambda_{f}, x, t_{f} ; t\right)}{\partial x} \\
& -\frac{\partial F_{3}\left(\lambda_{f}, x, t_{f} ; t\right)}{\partial t}+H\left(x,-\frac{\partial F_{3}\left(\lambda_{f}, x, t_{f} ; t\right)}{\partial x}, t\right)=0 \\
F_{4}: x_{f}= & \frac{\partial F_{4}\left(\lambda_{f}, \lambda, t_{f} ; t\right)}{\partial \lambda_{f}}, \quad x(t)=-\frac{\partial F_{4}\left(\lambda_{f}, \lambda, t_{f} ; t\right)}{\partial \lambda} \\
- & \frac{\partial F_{4}\left(\lambda_{f}, \lambda, t_{f} ; t\right)}{\partial t}+H\left(-\frac{\partial F_{4}\left(\lambda_{f}, \lambda, t_{f} ; t\right)}{\partial \lambda}, \lambda, t\right)=0
\end{aligned}
$$

These functions are all related by the Legendre transformations:

$$
\begin{gathered}
F_{2}\left(x_{f}, \lambda, t_{f} ; t\right)=F_{1}\left(x_{f}, x, t_{f} ; t\right)+\lambda^{T} x \\
F_{3}\left(\lambda_{f}, x, t_{f} ; t\right)=F_{1}\left(x_{f}, x, t_{f} ; t\right)-\lambda_{f}{ }^{T} x_{f} \\
F_{4}\left(\lambda_{f}, \lambda, t_{f} ; t\right)=F_{2}\left(x_{f}, \lambda, t_{f} ; t\right)-\lambda_{f}{ }^{T} x_{f}
\end{gathered}
$$

This means that among four possible forms of generating functions, only those two that are functions of both coordinates and momenta are well-defined at $t=t_{f}$. So we have to solve the HJ equation for $F_{2}$ or $F_{3}$ and then we can obtain solution of desired variables using Legendre transformation. Once the generating functions are obtained by solving HJ equation, one can solve any types of TPVBPs by simple function evaluations with various boundary conditions.

\subsection{Solution to two-point boundary-value problem}

By using the relations derived from canonical transformation, the solution to the TPBVP can be expressed by generating functions. For fixed terminal states problem, where the initial and terminal states are specified, $F_{3}$ -generating function can be directly used to find initial costates and final state:

$$
\begin{gathered}
\lambda_{0}=-\frac{\partial F_{3}\left(\lambda_{f}, x, t_{f} ; t\right)}{\partial x} \\
x_{f}=-\frac{\partial F_{3}\left(\lambda_{f}, x, t_{f} ; t\right)}{\partial \lambda_{f}}
\end{gathered}
$$

3.3 Robust terminal controller $\lambda_{f}$

In this section, we present some results on linear quadratic control with the worst case disturbance rejection based on Refs 12) and 13).
Consider the linear system defined on finite-time interval $\left[t_{0}, t_{f}\right]$ by

$$
\begin{gathered}
\dot{x}=A x+B_{1} w+B_{2} u \\
z=\left[\begin{array}{c}
C x \\
D u
\end{array}\right]
\end{gathered}
$$

It is assumed that the initial state $x\left(t_{0}\right)$ is possibly nonzero. Note that in the standard $H_{\infty}$ control, it is most often assumed that the initial state is zero. Khargonekar et al. introduced a worst case closed-loop performance measure which allows one to account for uncertainty in the initial condition $^{12)}$. For a fixed time $t_{f}>0$ and $X_{f}>0$, define the worst case closed-loop performance measure as

$$
J=\max \frac{\|z\|_{L^{2}\left[t_{0}, f_{f}\right]}^{2}+x^{T}\left(t_{f}\right) X_{f} x\left(t_{f}\right)}{\|w\|_{L^{2}\left[t_{0}, t_{f}\right]}^{2}+x_{0}^{T} x_{0}}
$$

where the maximum is take over all $x(0)=x_{0} \in \mathrm{R}^{n}$ and $w(t) \in L^{2}\left[t_{0}, t_{f}\right]$ such that $\|w\|_{L^{2}\left[t_{0} t_{j}\right.}^{2}+x_{0}^{T} x_{0}>0$. In terms of the performance measure (16), the $H_{\infty}$ synthesis problem can be introduced as

$$
\begin{gathered}
J^{*}=\min _{u} \max _{\left(x_{0}, w\right)} \frac{\|z\|_{L^{2}\left[t_{0}, f_{f}\right]}^{2}+x\left(t_{f}\right)^{T} X_{f} x\left(t_{f}\right)}{\|w\|_{L^{2}\left[t_{0}, t_{f}\right]}^{2}+x_{0}{ }^{T} x_{0}} \\
=\min _{u} \max _{\left(x_{0}, w\right)} \int_{t_{0}}^{t_{f}}\left(x^{T} Q x+u^{T} R u\right) d t+x\left(t_{f}\right)^{T} X_{f} x\left(t_{f}\right)
\end{gathered}
$$

where we write $\operatorname{diag}\left(Q^{1 / 2}, R^{1 / 2}\right)=[C, D]$. Here we consider the problem under the disturbance bounded by the windowed $L_{2}$-norm and norm bounded initial conditions, i.e., we wish to find the best control under the worst case disturbance and worst case initial conditions such that

$$
\begin{gathered}
\|w\|_{L^{2}\left[t_{0}, t_{f}\right]}^{2} \leq \rho \\
x_{0}^{T} x_{0} \leq \alpha^{2}
\end{gathered}
$$

The necessary conditions for the minimax problem lead to a Hamiltonian TPVBP. By using the variational approach, the minimax control law and the worst case disturbance to achieve

$$
\begin{aligned}
& J^{*}=\min _{u} \max _{\left(x_{0}, w\right)} \int_{t_{0}}^{t_{f}}\left(x^{T} Q x+u^{T} R u\right) d t+x\left(t_{f}\right)^{T} X_{f} x\left(t_{f}\right) \\
& =\max _{\left\|x_{0}\right\|=\alpha} x_{0}^{T} X\left(t_{0}\right) x_{0}+\mu \rho^{2} \\
& =\lambda_{m}\left[X\left(t_{0}\right)\right] \alpha^{2}+\mu \rho^{2}
\end{aligned}
$$

, where $\lambda_{m}$ is the maximal eigenvalue of $X(0)$, are given by

$$
\begin{aligned}
& u^{*}=-R^{-1} B_{2}^{T} \lambda \\
& w^{*}=-\frac{1}{\mu} B_{1}^{T} \lambda
\end{aligned}
$$

where $\lambda$ is a solution to the Hamiltonian system

$$
\left[\begin{array}{l}
x \\
\lambda
\end{array}\right]=\left[\begin{array}{cc}
A & -\left(B_{2} R^{-1} B_{2}^{T}-\frac{1}{\mu} B_{1} R^{-1} B_{1}^{T}\right) \\
-Q & -A^{T}
\end{array}\right]\left[\begin{array}{l}
x^{-} \\
\lambda
\end{array}\right.
$$

with boundary conditions 


$$
\left[\begin{array}{l}
x(0) \\
\lambda(0)
\end{array}\right]=\left\lfloor\begin{array}{l}
v^{-1} \lambda\left(t_{0}\right) \\
X_{f} x\left(t_{f}\right)
\end{array}\right\rfloor
$$

where $v^{-1}$ is equal to maximum eigenvalue $\lambda_{m}$ of $X(0)$ and $X(t)$ is given by

$$
\dot{X}+X A+A X-X\left(B_{2} R^{-1} B_{2}^{T}-\frac{1}{\mu} B_{1} B_{1}^{T}\right) X+Q=0, X\left(t_{f}\right)=X_{f}
$$

The Hamiltonian is given by

$$
H(x, \lambda, t) \equiv H\left(x, \lambda, u^{*}, w^{*}, t\right)
$$

\section{Application to Asteroid Deflection Mission}

\subsection{Equations of motion}

Consider a spacecraft subject to the central gravity field. The normalized equation of motion is described as:

$$
\ddot{\boldsymbol{r}}=-\frac{1}{r}+\boldsymbol{u}
$$

We consider the two-dimensional problem. The Lambert problem is solved to obtain nominal trajectory of the kinetic impactor spacecraft. At the terminal phase, linearized dynamics along a nominal trajectory are appropriate to describe the motion of spacecraft.

\subsection{Robust terminal controller based on generating function}

Here we assume that the initial state is nonzero and derive the robust controller to achieve desired terminal states under norm bounded initial conditions. Moreover we assume that we can observe all states and derive a state feedback controller. Let $x_{d}$ be the nominal ballistic trajectory described above. Define $\tilde{x}=x_{d}-x$ and linearized equation along the nominal trajectory is considered. To simplify the notation, we again use $x$ to describe the state of the linearized system. The linear quadratic control with worst case initial condition is used to keep the system as close as possible to the nominal trajectory. For this purpose, we describe the control (20) using second-order $F_{3}$ generating function:

$$
F_{3}=\frac{1}{2}\left[\begin{array}{l}
\tilde{x} \\
\lambda
\end{array}\right]^{T}\left[\begin{array}{ll}
F_{x x} & F_{x \lambda} \\
F_{\lambda x} & F_{\lambda \lambda}
\end{array} \rrbracket \tilde{x}_{-}^{-}\right.
$$

For the Hamiltonian system described by Eq. (22), HJ equation leads to the following differential equations :

$$
\begin{gathered}
\dot{F}_{x x}+F_{x x} A+A^{T} F_{x x}+F_{x x}\left(B_{2} R^{-1} B_{2}^{T}-\frac{1}{\mu} B_{1}^{T} B_{1}\right) F_{x x}-Q=0 \\
\dot{F}_{x \lambda}+A^{T} F_{x \lambda}+F_{x x}\left(B_{2} R^{-1} B_{2}^{T}-\frac{1}{\mu} B_{1}^{T} B_{1}\right) F_{x \lambda}=0 \\
\dot{F}_{\lambda \lambda}+F_{\lambda x}\left(B_{2} R^{-1} B_{2}^{T}-\frac{1}{\mu} B_{1}^{T} B_{1}\right) F_{x \lambda}=0
\end{gathered}
$$

and the terminal conditions are given by

$$
F_{x x}\left(t_{f}, t_{f}\right)=0, F_{x \lambda}\left(t_{f}, t_{f}\right)=-I, F_{\lambda \lambda}\left(t_{f}, t_{f}\right)=0
$$

from the identity transformation, $F_{3}\left(\lambda_{f}, x, t_{f} ; t_{f}\right)=x_{f}^{T} \lambda_{f}$. Using the relation (13), the costates terminal boundary condition can be expressed as

$$
\lambda\left(t_{f}\right)=X_{f} \tilde{x}_{f}=\left[\begin{array}{ll}
F_{\lambda x} & F_{\lambda \lambda}
\end{array}\right]\left[\begin{array}{c}
\tilde{x}_{f} \\
\lambda
\end{array}\right]
$$

Then

$$
\lambda(t)=\left[F_{x x}+F_{x \lambda}\left(I-X_{f} F_{\lambda \lambda}\right)^{-1} X_{f} F_{\lambda x}\right] x
$$

and $\mathrm{X}(0)$ is given by

$$
X(0)=F_{x x}\left(t_{f}, t_{0}\right)+F_{x \lambda}\left(t_{f}, t_{0}\right)\left(I-X_{f} F_{\lambda \lambda}\left(t_{f}, t_{0}\right)\right)^{-1} X_{f} F_{\lambda x}\left(t_{f}, t_{0}\right)
$$

Note that the control and the worst case initial condition is parametrized by terminal penalty function $X_{f}$.

\subsection{Numerical simulations}

The robust terminal controller is applied to the terminal guidance control of kinetic impactor spacecraft. One can obtain the nominal transfer trajectory to the asteroid by solving Lambert's problems. Here we assume the nominal trajectory is given for a fictitious asteroid. The nominal trajectory is shown by green line in Fig. 1 and that of target asteroid is shown by dotted line. In the figure, the terminal phase starts from the origin and the trajectories are shown in non-dimensional unit. The trajectory of the fictitious asteroid which starts from $\boldsymbol{r}=(x, y)=(0.062,0.073)$ is given by dotted line. The robust terminal controller is applied to change the terminal intercept angle. The controlled trajectories given by generating functions approach are shown in blue lines of Figure 2. Note that all trajectories are obtained by one generating function, i.e., we can estimate the fuel to change the intercept angle immediately. Figure 3 shows total $\Delta v$ requirements vs. impact angle with varying the non-dimensional terminal relative velocity where the impact angle is measured from tangential direction.

Figure 4 shows the distribution of final position error of 100 randomly chosen disturbances with $\alpha, \rho=0.01$. The red and blue points show the position errors of LQR controllers with and without disturbances and green one shows that of robust controller. We can see that the robust controller suppresses the final state error.

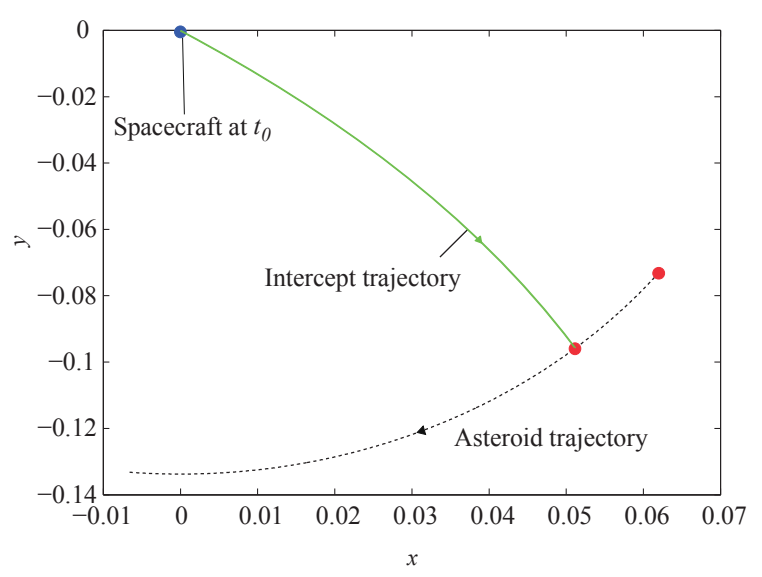

Fig. 1. Nominal trajectory. 


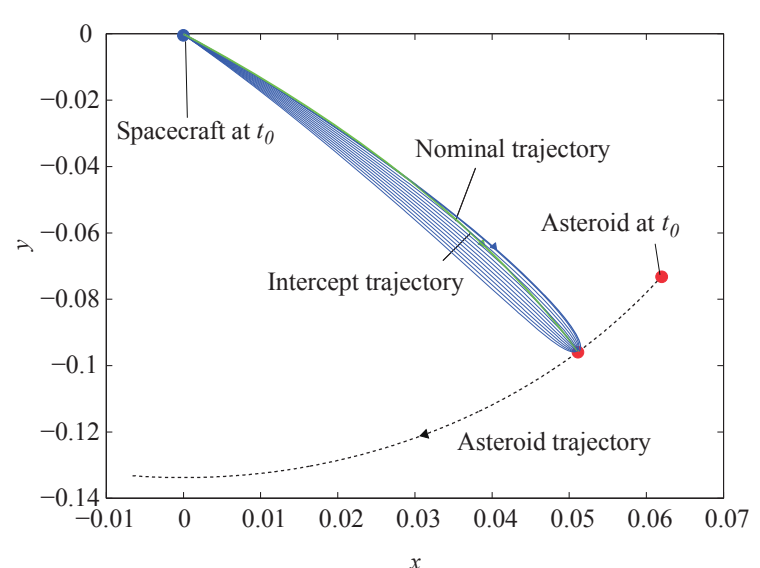

Fig. 2. Nominal and controlled trajectories.

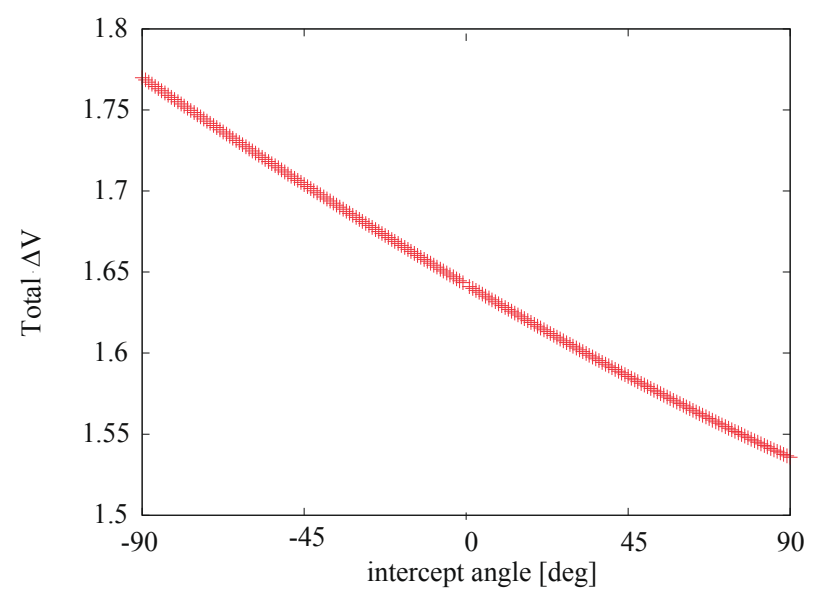

Fig. 3. Total $\Delta v$ requirements vs. impact angle.

\section{Conclusions}

The trajectory design associated with the deflection of potentially hazardous asteroid (PHA) was considered. The robust trajectory design for kinetic impactor spacecraft, including the uncertainty of orbital elements and options to use the low-thrust acceleration was considered. Moreover, the asteroid deflection mission with a controlled intercept angle was considered. We employed the generating functions approach and investigated its application to robust terminal guidance control using low-thrust acceleration. The advantage of using our approach was that performance by changing various parameters was easy to estimate. Future study includes the estimate of the closest approach distance and the application to real asteroids.

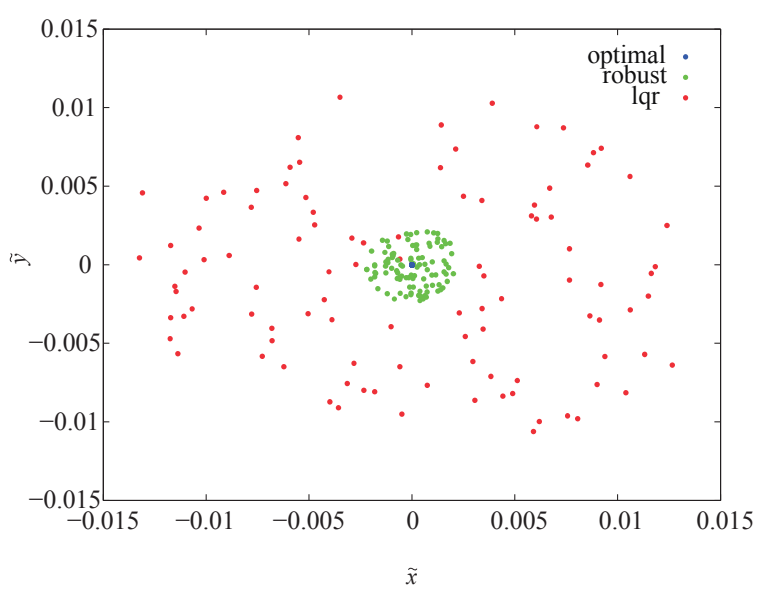

Fig. 4. Total $\Delta v$ requirements vs. impact angle

\section{References}

1) Committee to Review Near-Earth Object Surveys and Hazard Mitigation Strategies, National Research Council, Defending Planet Earth: Near-Earth Object Surveys and Hazard Mitigation Strategies, Final Report, The National Academies Press, January, (2010).

2) Conway, B.A.: Two-body optimization for deflecting Earth-crossing asteroids, Journal of Guidance, Control, and Dynamics, 24 (2001), pp. 1035-1037.

3) Park, S.Y. and Ross, I.M.: Two-body optimization for deflecting Earth-crossing asteroids, Journal of Guidance, Control, and Dynamics, 22 (1999), pp. 415-420.

4) Park, S.Y. and Mazanek, D.D.: Mission functionality for deflecting earth-crossing asteroids/comets, Journal of Guidance, Control, and Dynamics, 26 (2003), pp. 734-742.

5) Park, S.Y. and Choi, K.H.: Optimal low-thrust intercept/rendezvous trajectories to earth-crossing objects, Journal of Guidance, Control, and Dynamics, 28 (2005), pp. 1049-1055.

6) Izzo, D.: Optimization of interplanetary trajectories for impulsive and continuous asteroid deflection, Journal of Guidance, Control, and Dynamics, 30 (2007), pp. 401-408.

7) Vasile, M.: Optimal Impact Strategies for Asteroid Deflection, Journal of Guidance, Control, and Dynamics, 31 (2008), pp. 858-872.

8) Guibout, V.M. and Scheeres, D.J.: Spacecraft formation dynamics and design, Journal of Guidance Control and Dynamics, 29 (2006), pp. 121-133.

9) Park, C. and Guibout, V. M. and Scheeres, D. J.: Solving Optimal Continuous Thrust Rendezvous Problems with Generating Functions, Journal of Guidance, Control, and Dynamics, 29 (2006), pp. 321-331.

10) Bando, M. and Yamakawa, H.: A New Optimal Orbit Control for Two-Point Boundary-Value Problem Using Generating Functions, AAS/AIAA Space Flight Mechanics Meeting, (2009), 09-117.

11) Goldstein, H.: Classical Mechanics, Addison-Wesley, Reading, (1980).

12) Khargonekar, P., Nagpal, K. and Poolla, K.: H-infinity Control with Transients, SIAM Journal on Control and Optimization, 29 (1991), pp. 1373-1393.

13) Lu, W.W., Balas, G.J. and Lee, E.B.: Linear quadratic performance with worst case disturbance rejection, International Journal of Control, 73 (2000), pp. 1516-1524. 